\title{
PUBLICATION 1012
}

30.4

212

1012

957

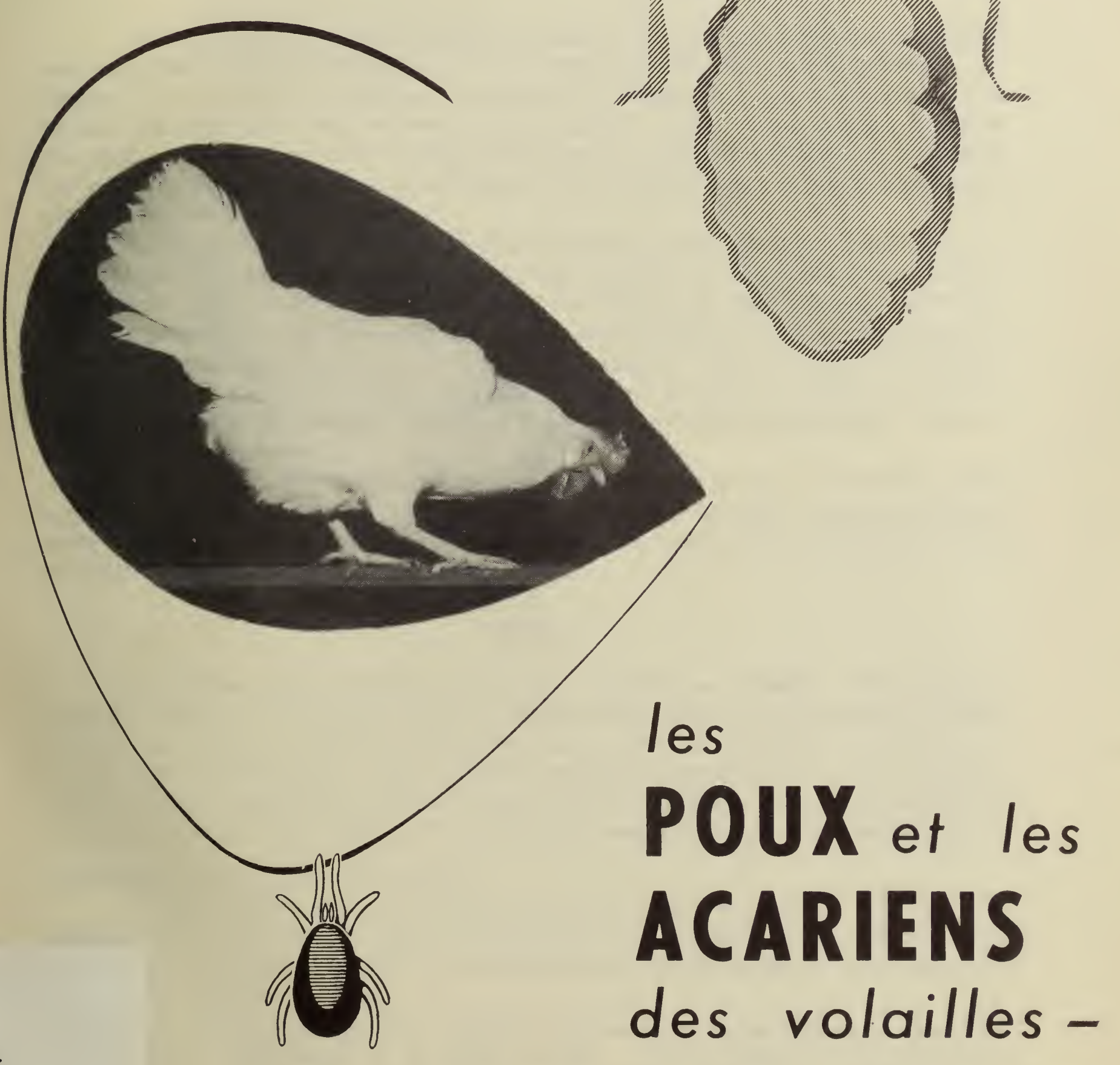

comment les detruire

Par P.E. MORRISON

et R.P. THOMPSON

MINISTERE DE L'AGRICULTURE DU CANADA 



\title{
LES POUX ET LES ACARIENS DES VOLAILLES COMMENT LES DÉTRUIRE
}

\author{
Par \\ P.E. Morrison et R:P. Thompson \\ Laboratoire d'entomologie, Guelph (Ontario)
}

$\mathrm{Au}$ Canada, plusieurs espèces de poux et d'acariens sont très nuisibles aux volailles. Les infestations par ces insectes endommagent le plumage, retardent la croissance, réduisent la vitalité et entrainent une diminution de la production d'oeufs; elles peuvent meme causer la mort. Les oiseaux înfestés mangent inoins ou, par contre, consomment une plus grande quantité de nourriture par suite de l'épuisement de leur vigueur; ils s'affaiblissent et deviennent ainsi plus prédisposés aux maladies.

Les poux et les acariens se reproduisent rapidement par temps chaud et peuvent se rencontrer en grand nombre. Ils peuvent se propager d'une ferme à l'autre par l'entremise de volailles errant en liberté ou nouvellement achetées, d'oiseaux sauvages, de cageots ou de cages å volailles, ou sur les vetements de personnes manutentionnant des volailles infestees. Les mesures sanitaires appropriěes et d'autres moyens préventifs, comme la pose de grillages aux fenêtres des poulaillers afin d'empêcher l'entrěe des oiseaux sauvages, aident à prévenir les infestations.

Etant donné que les poux sont des insectes, tandis que les acariens sont plus étroitement apparentés aux araigněes, les deux groupes varient beaucoup entre eux quant à l'apparence, le cycle évolutif, les moeurs et les moyens de lutte, de sorte qu'ils sont étudiěs séparěment.

\section{POUX}

On trouve sept espe̊ces de poux des volailles au Canada. Ces poux, qui sont des insectes broyeurs ne sucent pas le sang. Ils se nourrissent de plumes, de poil, des écailles libres et des gales sur la peau. Ils nuisent aux oiseaux en les irritant constamment. Les volailles fortement infestěes ont l'air abattues, elles trainent leurs ailes, et leurs plumes sont en désordre. L'examen périodique des oiseaux aide å découvrir les infestations avant qu'elles deviennent nuisibles.

Apparence, cycle évolutif et moeurs

Le pou adulte est brun jaunatre ou noir et mesure, selon l'espe̊ce, d'un huitième å un seizie̊me de pouce de longueur; il a le corps aplati et la tete large et arrondie. Ses parties buccales comprennent deux mandibules ou dents pointues qui servent a broyer.

Les oeufs sont petits, mesurant moins d'un trente-deuxième de pouce de diamètre, blanchatres et de forme ovale. On les trouve généralement en grappes ou masses grisatres attachées aux barbes des plumes. (Figure 1). On les rencontre sur diverses parties du corps, selon l'espèce. Les oeufs éclosent de deux å quinze jours après la ponte. En géněral, ils n'éclosent pas sur les plumes enlevées du corps de l'oiseau. Les nymphes nouvellement écloses ressemblent aux adultes, sauf qu'elles sont plus petites et plus pàles. Elles muent, ou jettent leur peau, plusieurs fois avant de se développer en insectes adultes, ce qui se produit généralement dans moins de trois semaines. 


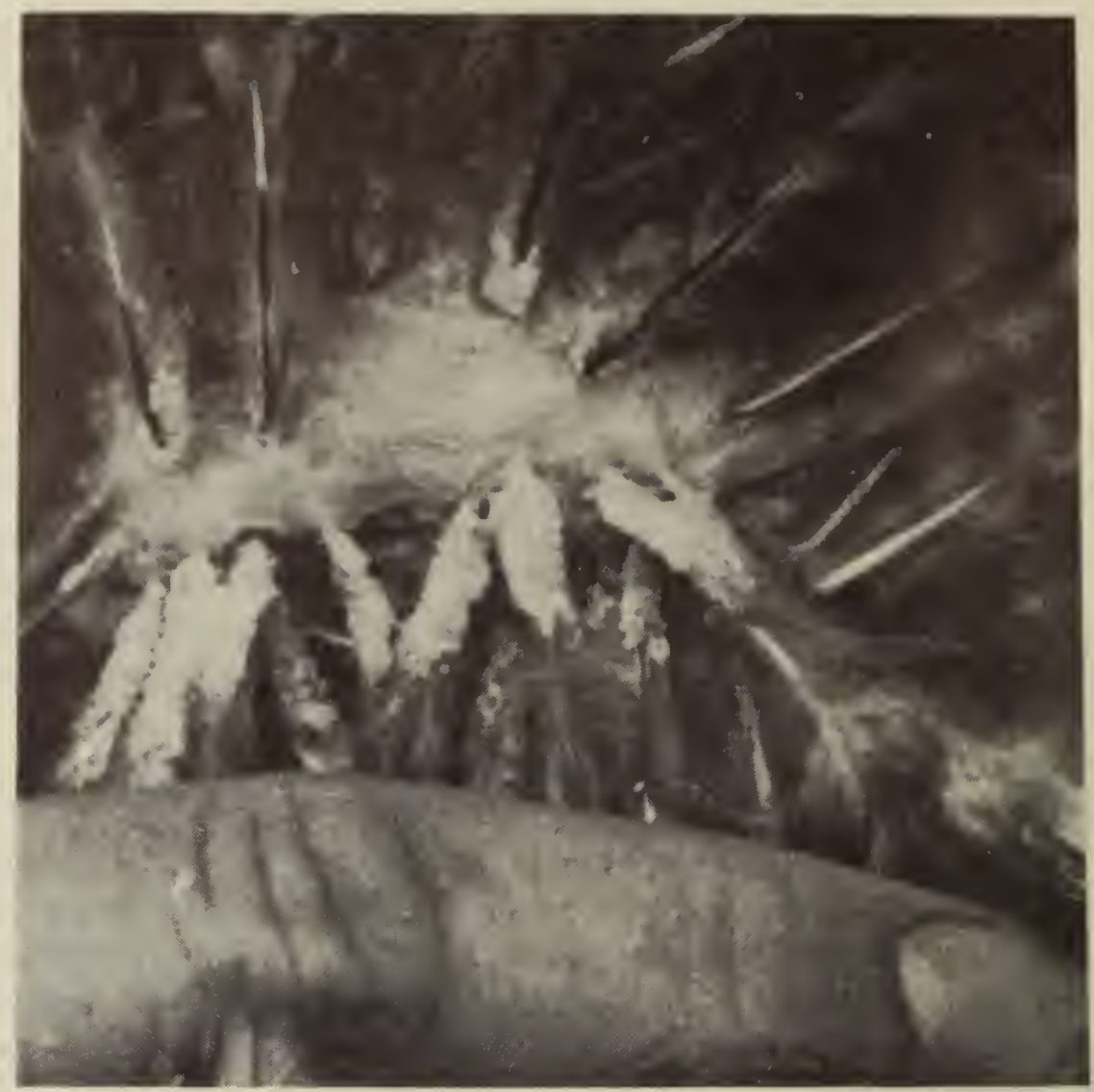

Figure 1. - Masses d'oeufs du pou du corps des volailles attachées aux plumes près de l'anus.

Les poux passent toute leur vie sur les oiseaux et ne peuvent survivre ailleurs pendant plus de sept à dix jours. Ils ne vivent sur les etres humains qu'un jour ou deux. Ils se reproduisent le plus rapidement en été, mais meme en hiver on trouve toutes les phases du cycle évolutif sur les oiseaux, soit des o eufs, des nymphes et des adultes. Le nombre de poux s'accroit rapidement en général, une fois qu'ils se sont implantés. En séparant les plumes d'un oiseau infesté, on peut voir les poux courir sur la peau ou collés aux plumes.

\section{Espèces de poux attaquant les volailles}

Les trois espèces les plus répandues sont le pou du corps des volailles, Menacanthus stramineus (Nitz.), le pou des volailles, Menopon gallinae (L.), et le petit pou des poulets, Goniocotes gallinae (DeG.). Le pou des ailes, Lipeurus caponis (L.), et le pou de la tete des volailles, Cuclotogaster heterographus (Nitz.), sont aussi très répandus dans certaines parties du pays. Le pou géant des volailles, Goniodes gigas (Tasch.), et le pou brun des volailles, Goniodes dissimilis Denny, sont des especes moins répandues. Les volailles peuvent souvent être infestées par deux espèces ou plus en mème temps.

Pou du corps des volailles

Le pou du corps est probablement celui qui cause le plus d'irritation. Il mesure environ un huitième de pouce de long. Chez les volailles adultes. il se rencontre sur la peau autour de l'anus et souvent sur les cotés du corps. Chez les jeunes poulets, c'est sur le dos qu'on le voit en plus grand nombre. Souvent, une infestation grave peut causer le rougissement marqué de la peau. Meme chez les oiseaux légèrement ou modérément infestés, on peut voir les poux cherchant à se cacher lorsque les plumes sont séparées. Les masses d'oeufs se trouvent gěněralement sur les plumes autour de l'anus. 
Pou des volailles

Ce pou ressemble à celui du pou du corps des volailles, mais il est plus petit, mesurant environ un seizième de pouce de long. On le rencontre surtout sur les cotés du corps, sur les cuisses et autour de l'anus, attaché aux plumes, la tete en bas. Lorsque les oiseaux sont fortement infestés, on en trouve aussi sur les plumes du dos. Les poux cherchent à se rendre jusqu'à la peau lorsque les plumes sont séparées. Les oeufs, attachěs à la base des plumes, éclosent au bout de deux semaines ou moins.

Seuls les vieux oiseaux sont exposés à l'infestation par le pou des volailles, probablement parce que les plumes des jeunes oiseaux ne sont pas complètement développées. Les canards, les dindons et les pintades peuvent aussi etre infestés s'ils sont logés avec des volailles infestées.

Petit pou des poulets

On trouve cette espèce chez les oiseaux les plus duveteux. C'est un insecte pale, rondelet, mesurant environ un vingtième de pouce de long; le petit pou des poulets, qui se rencontre rarement en grand nombre, ne semble pas etre nuisible.

Pou des ailes

Cet insecte grele, gris ou noir, mesure environ un dixieme de pouce de long; on le trouve gènéralement attaché aux rémiges primaires (Figure 2). On le rencontre parfois sur les plumes du cou.

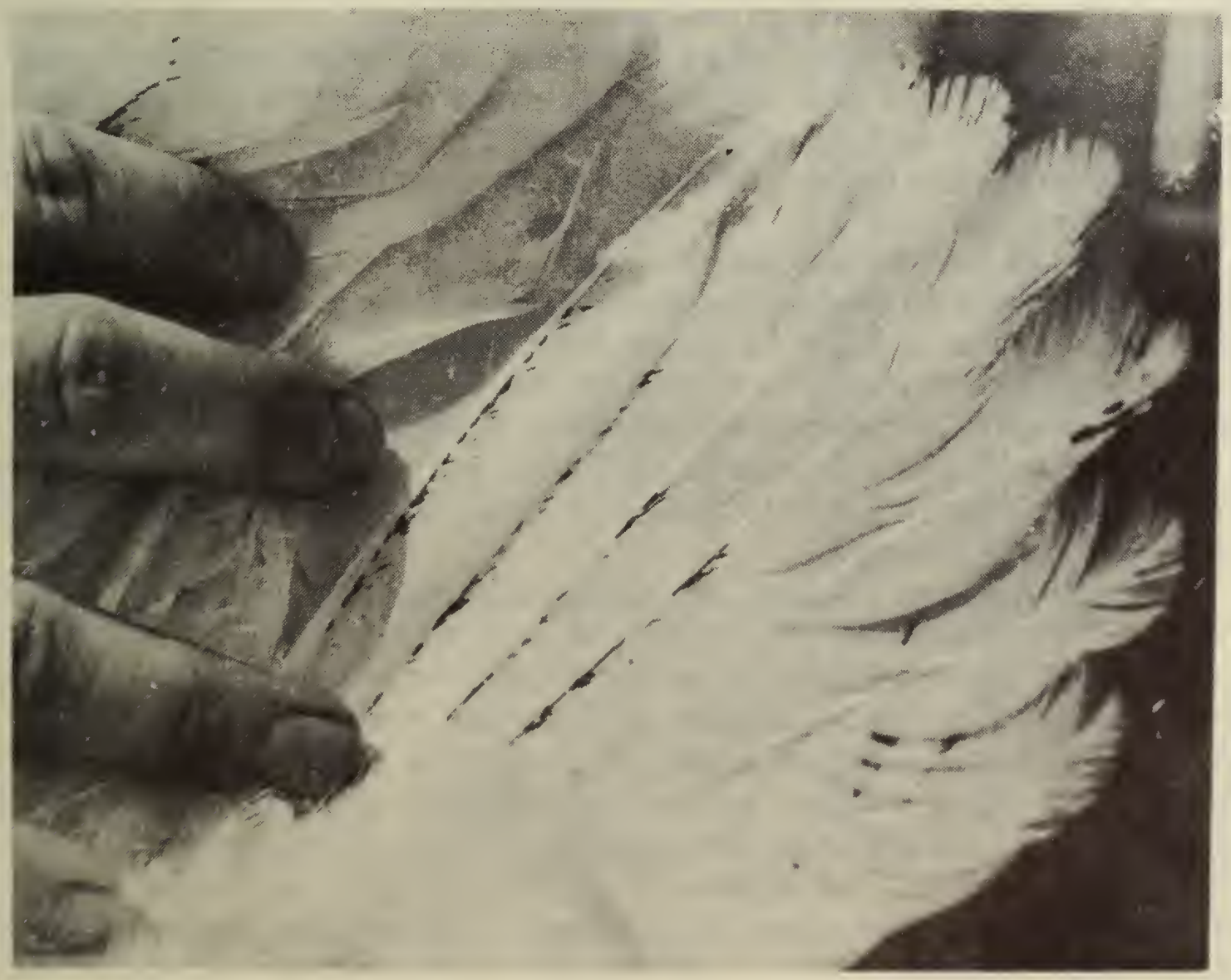

Figure 2. Poux des ailes attachés aux barbes des rémiges primaires. 
Pou de la tête des volailles

Cette espèce se rencontre généralement en plus grand nombre sur les jeunes poulets. Les poux, gris foncé, mesurent environ un dixième de pouce de long. Ils se laissent pendre, la tete en bas, à la base des plumes sur le dessus de la tete de l'oiseau. Les oeufs sont collés aux barbes du duvet ou des petites plumes sur la tete ou le cou des poulets; ils éclosent au bout de 10 jours environ. Le pou de tete passe de la poule couveuse aux poussins, après quoi il ne se déplace que très peu. Il peut etre nuisible aux poussins et aux dindonneaux fortement infestés.

Autres espèces

Le pou géant des volailles, dont le corps est large, de couleur gris fumée à noire, mesure environ un huitième de pouce de long. On le rencontre sur les plumes de diverses parties du corps. Le pou brun des volailles, plus petit que l'espèce précédente, est brun rougeatre et se cantonne sur les plumes du corps.

Moyens de lutte

Une seule application de malathion est efficace contre toutes les espèces de poux des volailles. Appliquez-le en poudre (4 p.100) sur la litière du plancher, les juchoirs et la litière des nids, à raison de 1 livre par 40 pieds carrés. C'est là le plus simple moyen d'application et on le recommande surtout par temps froid. Epandez la poudre à la volée au moyen d'une poudreuse mécanique, d'une boite à couvercle perforé ou avec la main gantée. Par temps chaud, on peut appliquer une pulvérisation de malathion 3 p.100. Préparez cette pulvérisation en mélangeant parfaitement $2 \frac{1}{2}$ chopines d'un produit concentré émulsifiable 50 p.100, ou 6 livres d'une poudre mouillable 25 p.100 dans 5 gallons d'eau. Appliquez cette solution avec un pulvérisateur à jardin ou à moteur sur la litière du plancher, les juchoirs et la litière des nids, en quantité suffisante pour les mouiller. Pulverisez le côté ainsi que le dessus des batons des juchoirs. Le Lindane pulvérisé sous forme d'émulsion d'eau 0.5 p.100 est efficace également. Il n'est pas nécessaire de faire sortir les oiseaux du parquet durant l'un ou l'autre de ces traitements.

Une autre méthode consiste à poudrer avec du malathion tous les oiseaux infestěs du troupeau ou chaque oiseau séparément. Pour le traitement du troupeau, appliquez une poudre 4 p.100 au moyen d'une poudreuse rotative ou d'une boite à couvercle perforé, à raison de 1 livre par 100 oiseaux; pour traiter les oiseaux séparément, poudrez au moyen d'une poudreuse à soufflet, à raison de 1 livre par 150 oiseaux. Deux coups de soufflet par oiseau suffisent si la poudre est dirige̊e vers l'anus et la poitrine. Le poudrage est des plus efficaces pour les oiseaux en batteries et sur parcours.

Un autre moyen de lutte, moins sür, consiste à peinturer les juchoirs. Appliquez sur les juchoirs, au pinceau ou avec un pulverisateur, une émulsion de malathion 3 p.100 ou de la poudre mouillable en suspension, à raison de 20 nnces liquides par 50 pieds linéaires des juchoirs. De plus, pulvérisez du malathion 1 p.100 sur la litiere des nids de façon à la mouiller. Ou appliquez du Lindane de la meme manière, soit en pulvérisation 1 p.100 sur les juchoirs à raison de 20 onces liquides par 200 pieds linéaires, et en pulvérisation 0.5 p.100 sur la litière des nids. Le sulfate de nicotine 40 p.100 peut aussi etre utilisé pour peinturer les juchoirs dans les batiments bien ventilés lorsque la température intérieure dépasse $70^{\circ} \mathrm{F}$. Appliquez 16 onces liquides par 200 pieds des juchoirs.

Les recommandations relatives aux moyens de lutte sont indiquées au tableau 1. 


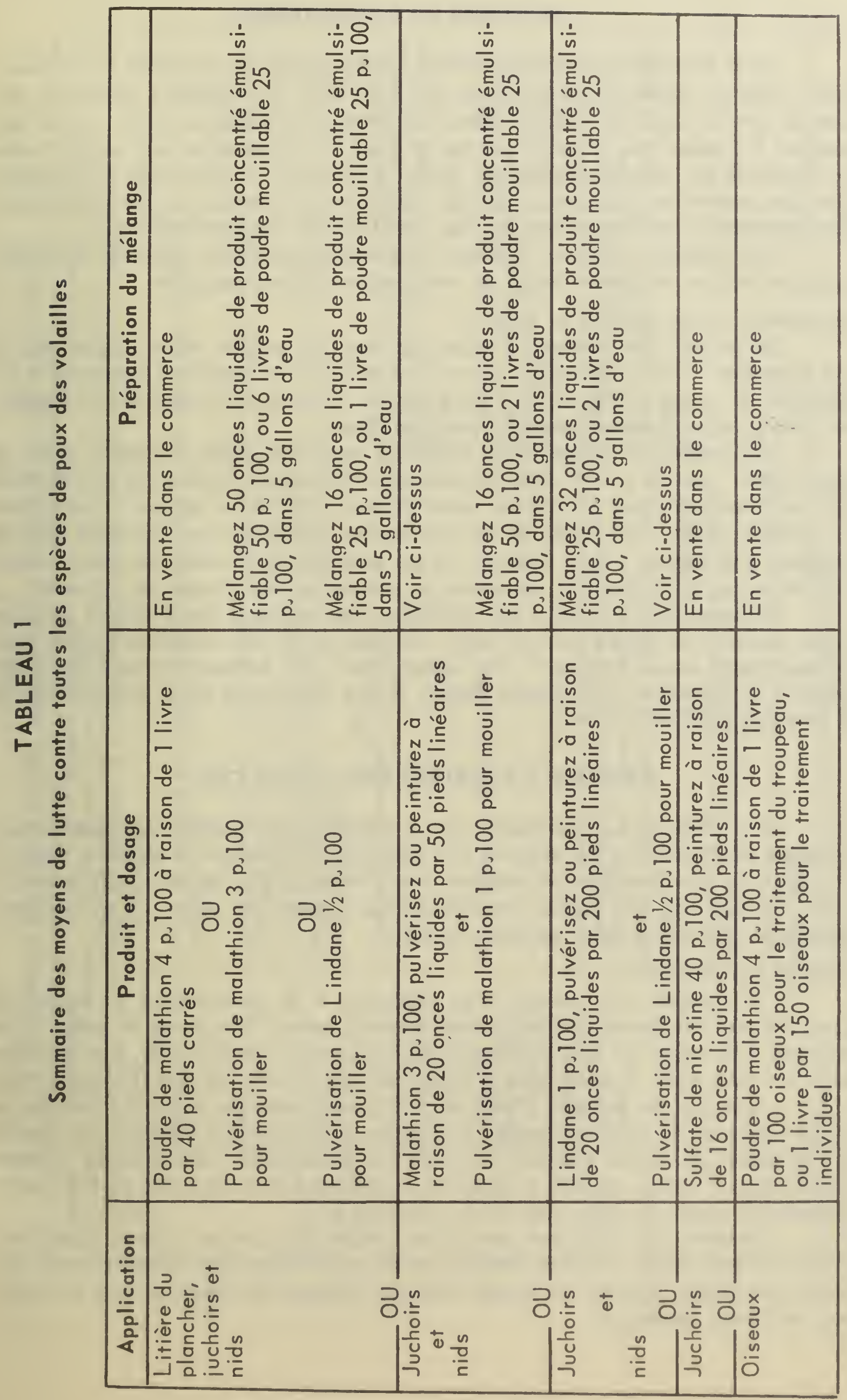




\section{ACARIENS OU DERMANYSSES}

Trois espèces d'acariens infestent généralement les volailles au Canada. Deux espèces vivent continuellement sur leur hote; la troisième n'attaque les oiseaux que la nuit, se cachant dans les crevasses durant le jour. Tous les acariens infestant les volailles sucent le sang. L'infestation par les acariens se manifeste de diverses maniêres, selon le troupeau; la nervosité et l'anémie sont des symptomes typiques. Il faut examiner les oiseaux et les poulaillers régulièrement et minutieusement si l'on veut dépister les infestations.

Les acariens peuvent propager la pneumoencéphalite (maladie de Newcastle) et on les soupçonne aussi de transmettre d'autres maladies.

Apparence, cycle évolutif et moeurs

La couleur des acariens adultes, qui ont huit pattes, varie de gris pale a noir rougeatre. Ces insectes mesurent de moins d'un soixante-quatrième à un trentieme de pouce de longueur. Leurs parties buccales sont pointues et aigues, de sorte qu'elles peuvent percer la peau de l'hote.

Les oeufs des acariens des volailles sont tres petits, de forme ovale et blanc perle. On les trouve dans les colonies d'acariens adultes, et ils éclosent au bout d'une semaine. L'acarien nouvellement éclos a sıx pattes et ressemble a l'insecte adulte. Au cours de sa croissance, l'acarien mue plusieurs fois et acquiert huit pattes. Les endroits où les acariens se groupent en grand nombre sont sales, qu'il s'agisse de crevasses du parquet ou des plumes de l'oiseau.

Contrairement aux poux des volailles, les acariens des volailles peuvent mieux survivre en dehors de leur hote. Les moeurs de ces acariens varient considérablement selon l'espèce. Par temps froid, une infestation peut demeurer inactive et inaperçue. Par temps chaud, toutes les phases sont plus actives et le cvcle évolutif plus court.

\section{ESPÈCES D'ACARIENS DES VOLAILLES}

Les acariens qui infestent le plus souvent les volailles au Canada sont l'acarien des volailles (ou acarien des juchoirs), Dermanyssus gallinae (DeG.), le liponysse des volailles (ou acarien des plumes), Bdellonyssus sylviarum (C. \& F.), le sarcopte des pattes, Knemidokoptes mutans (R. \& L.), et le sarcopte déplumant, Knemidokoptes gallinae (Raill.).

Acarien des volailles

Cet insecte, qui mesure d'un trentième à un quarantième de pouce de long, est plus facilement visible immédiatement après s'etre gorgè de sang, alors qu'il est d'un rouge vif. Par la suite, il devient rouge foncé ou noir. Les acariens qui n'ont pas sucé de sang sont gris et. par conséquent, plus difficiles â voir.

L'acarien des volaille s'attache à l'oiseau durant la nuit, suce le sany et cause beaucoup de malaise. Durant la journée, il vit dans les joints et les fentes des juchoirs, les boites à nids et les murs des poulaillers. Ces endroits présentent un aspect "poivre et sel" à cause de la présence des acariens, de leur peaux blanchatres rejetées, des oeufs et des fientes.

L'acarien des volailles peut vivre quatre ou cinq mois sans nourriture, meme par les temps les plus froids. Si la poussinière est chauffěe avant d'y mettre les jeunes oiseaux, on pourra voir les acariens se déplacer dans le voisiuage de leurs cachettes. 


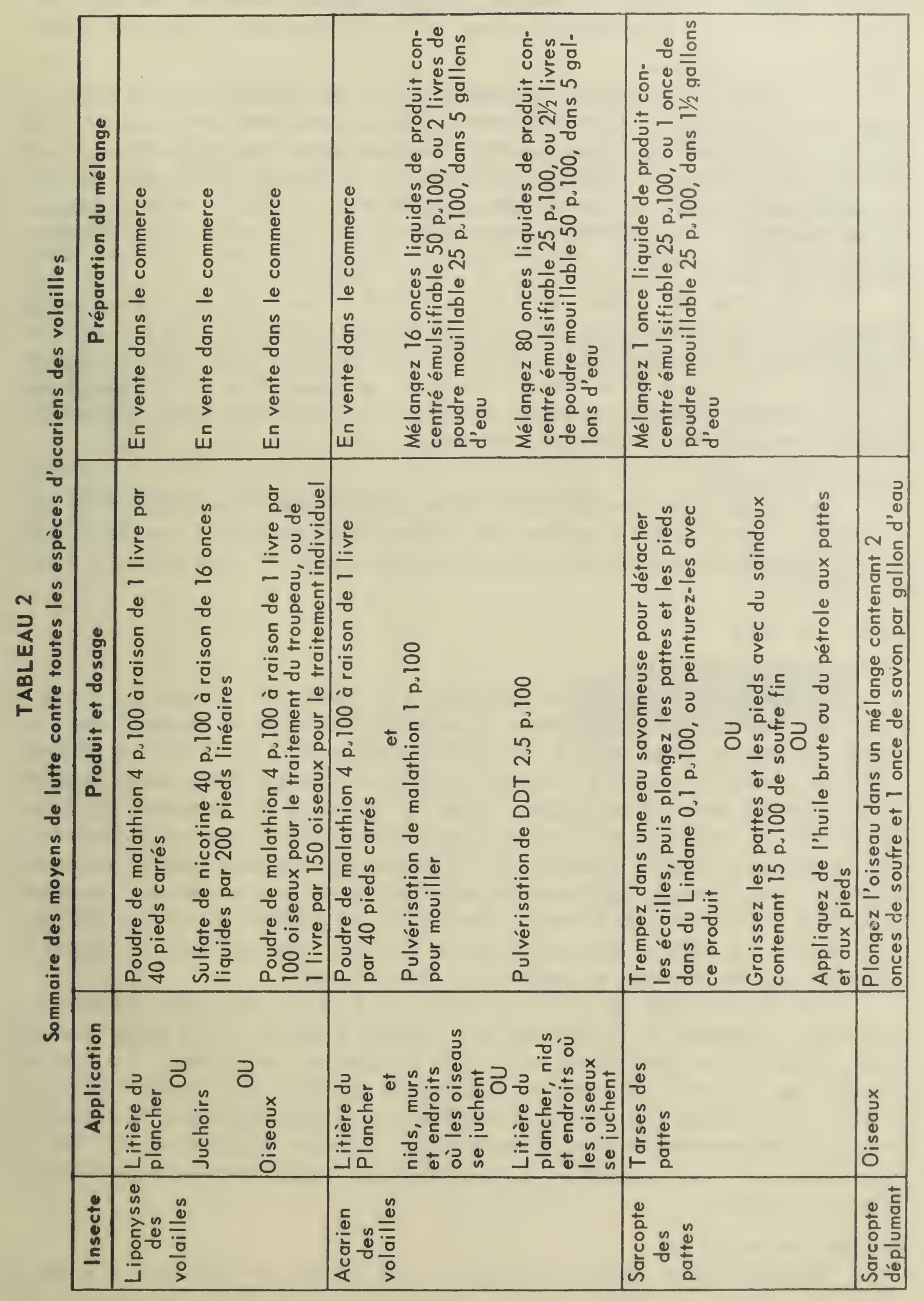


Les poulets semblent etre l'hote préféré, mais les acariens peuvent s'attacher a d'autres oiseaux et animaux domestiques et sauvages, et aux etres humains. Ainsi, les infestations peuvent se transmettre d'un troupeau à l'autre.

\section{Lyponysse des volailles}

Cet insecte, qui mesure d'un quarantième à un cinquantième de pouce de long, ressemble à l'acarien des volailles. Ses moeurs diffèrent de celles de ce dernier; le liponysse vit constamment sur l'oiseau et se tient généralement près de l'anus, de la queue et du cou. Les fientes et les oeufs des acariens donnent une apparence grisatre et poivrée aux plumes de ces régions. Une infestation par ces insectes se constate par leur présence sur les oeufs et la litiere des nids.

Sarcopte des pattes

Le sarcopte des pattes est à peine visible à l'oeil nu. Il s'enfonce dans la chair sous les écailles des pattes des volailles où il suce le sang et entraine l'épaississement des écailles. Il se forme des taches rouges dans les régions infestees, et il peut y avoir effusion de sang. Au bout d'un certain temps, les oiseaux infestés peuvent se mettre à boiter. On rencontre aussi cet acarien autour de la tête et de la crete de l'oiseau.

\section{Sarcopte déplumant}

Cet insecte est à peu près de la même grosseur que le sarcopte des pattes. Il s'enfonce dans la peau à la base des plumes de la croupe, du dos, de la tête, de l'abdomen et des pattes. Souvent les oiseaux arrachent leurs plumes à cause de l'irritation.

\section{Moyens de hutte}

Liponysse des volailles

Appliquez à la litière du plancher une poudre de malathion 4 p.100 à raison de 1 livre par 40 pieds carrés. Epandez-la å la volée au moyen d'une poutdreuse mécanique, d'une boite à couvercle perforé ou à la main munie d'un gant. Une autre méthode consiste à poudrer les oiseaux infestés dans tout le troupeau ou séparément, avec du malathion. Pour le traitement du troupeau, appliquez une poudre de malathion 4 p.100 au moyen d'une bolte à couvercle perforé ou d'une poudreuse rotative, à raison de 1 livre par 100 oiseaux; pour le traitement individuel des oiseaux, appliquez le malathion avec une poudreuse a soufflet, à raison de 1 livre par 150 oiseaux. Deux coups de soufflet par oiseau suffisent si la poudre est dirigée vers l'anus et la poitrine. Le poudrage est des plus efficaces pour les oiseaux en batteries ou sur parcours.

Cet insecte peut aussi etre réprimé par l'emploi de sulfate de nicotine 40 p.100. Peinturez ce produit sur les juchoirs à raison de 16 onces par 200 pieds linéaires une demi-heure avant que les poules se juchent. La chaleur dégagée par le corps de l'oiseau vaporise la nicotine qui s'infiltre dans les plumes. Pour obtenir les meilleurs résultats possibles, appliquez le traitement lorsque la température de l'air dépasse $70^{\circ} \mathrm{F}$. Répétez l'application au bout de deux semaines. Les poulaillers doivent etre bien ventilès lorsqu'on se sert du sulfate de nicotine.

\section{Acarien des volailles}

Appliquez à la litière du plancher une poudre de malathion 4 p.100 à raison de 1 livre par 40 pieds carrés, et en outre pulvérisez une émulsion de malathion 1 p. 100 ou de la poudre mouillable en suspension sur les nids, les murs et les endroits destiněs au juchage en quantité suffisante pour les mouiller. 
Ou encore appliquez à la litière du plancher, aux nids et aux juchoirs une pulvĕrisation de DDT $2 \frac{1}{2} 2$ p. 100 , préparée en mélangeant $2 \frac{1}{2}$ livres de poudre mouillable de DDT 50 p.100 dans 5 gallons d'eau. Le malathion donne une rěpression plus rapide que que le $\mathrm{DDT}$.

Sarcopte des pattes

Faites tremper les tarses des pattes et les pieds des oiseaux dans une eau savonneuse jusqu'à ce que les écailles se détachent. Puis plongez les pattes et les pieds dans une émulsion de Lindane 0.1 p. 100 ( 1 partie par 1000), préparée avec un produit concentré émulsifiable, ou peinturez-les avec cette solution. Un autre moyen consiste à graisser les pattes et les pieds avec du saindoux contenant 15 p.100 de soufre finement moulu (microfin). Ou encore plongez la partie inférieure des pattes dans de l'huile bruite, répétant le traitementaubout d'un mois si les écailles tordues ne se sont pas détachées.

Sarcopte déplumant

Plongez les oiseaux dans un mélange préparé avec 2 onces de soufre et 1 once de savon ou de détergent dans un gallon d'eau chaude. Répétez le traitement toutes les 3 ou 4 semaines.

Les moyens de lutte recommandés sont résumés dans le tableau 2.

Pour de plus amples renseignements veuillez écrire à la Sectionde l'entomologie médicale et véterinaire, édifice du Service des sciences, Ottawa (Ont.), ou au Laboratoire de l'entomologie de votre province.

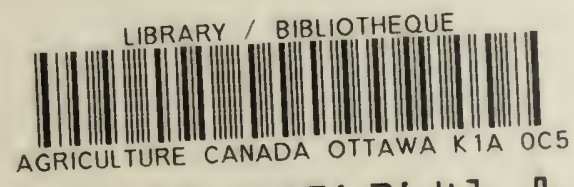

39073 0006264l 8 
EDMOND CLOUTIER, C.M.G., O.A., D.S.P

IMPRIMEUR DE LA REINE ET CONTRÔLEUR DE LA PAPETERIE OTTAWA, 1957 